\title{
Locally probing the screening potential at a metal-semiconductor interface
}

\author{
Ying Jiang,,${ }^{1,2, *}$ J. D. Guo, ${ }^{1}$ Ph. Ebert ${ }^{3}$ E. G. Wang, ${ }^{1}$ and Kehui $\mathrm{Wu}^{1}$ \\ ${ }^{1}$ Institute of Physics, Chinese Academy of Sciences, Beijing 100080, China \\ ${ }^{2}$ Department of Physics and Astronomy, University of California, Irvine, California 92697-4575, USA \\ ${ }^{3}$ Institut für Festkörperforschung, Forschungszentrum Jülich GmbH, 52425 Jülich, Germany
}

(Received 15 November 2009; published 21 January 2010)

\begin{abstract}
The screened Coulomb potential of a point charge located at buried $\mathrm{Ag} / \mathrm{Si}$ interface was quantitatively investigated using scanning tunneling microscopy and spectroscopy at $77 \mathrm{~K}$, through $\mathrm{Ag}(111)-1 \times 1$ films epitaxially grown on a $\mathrm{Si}(111)-\sqrt{3} \times \sqrt{3}-\mathrm{Ga}$ substrate. On top of the Ag films, we succeeded to image the two-dimensional screening potential around the individual charged $\mathrm{Si}$ dopants located at the $\mathrm{Ag} / \mathrm{Si}$ interface. The interface screening length was derived experimentally, which agrees well with a semimicroscopic theoretical model.
\end{abstract}

DOI: 10.1103/PhysRevB.81.033405

PACS number(s): 68.37.-d, 73.20.-r, 73.21.Fg

Screening is one of the key issues in solid state physics, ${ }^{1}$ which modifies the electron-electron interaction and excitation, and plays important roles in various physical properties of solid state materials, e.g., the nonlinear optical response of semiconductors $^{2}$ and the electron-phonon coupling in high $T_{c}$ superconductor. ${ }^{3}$ It is particularly remarkable that screening effects on a metallic surface can be directly visualized using scanning tunneling microscopy (STM) and spectroscopy (STS) at nano- and subnanometer scale, for examples, the Friedel oscillations of the charge density around defects on metal surfaces, ${ }^{4}$ the potential fluctuations in two-dimensional (2D) system, ${ }^{5}$ and the screened Coulomb potential around the dopant atoms on cleaved semiconductor surfaces. ${ }^{6}$ On the other hand, so far, very little has been known about the screening at interfaces, although it is obviously a more important issue for realistic device applications. The main difficulty lies in that STM is basically a surface sensitive technique, ${ }^{7}$ and the interface information is blocked due to the extremely short screening length in metals. ${ }^{1}$ Besides, it is nontrivial to prepare an atomically sharp interface, with screening centers (dopants, impurities) exactly located at the interface plane, such that quantitative study could be carried out.

In this Brief Report, we have realized a system ideally suitable for studying the interface screening in atomic scale. The system consists of a sandwiched structure: Ag film/2D semiconducting $\sqrt{3} \times \sqrt{3}-\mathrm{Ga}$ layer/Si substrate, where the positively charged dopants $(\mathrm{Si})$ are exactly located at the $\mathrm{Ag} / \mathrm{Si}$ interface. We report on a direct visualization of the screening potential around individual dopant atoms at such a buried metal-semiconductor interface. The screening length was quantitatively derived from the experiment, which agrees well with a semimicroscopic theoretical model.

The experiments were carried out in a LT-STM (Omicron) system in ultrahigh vacuum $\left(p<1 \times 10^{-11}\right.$ mbar $)$. A clean Si(111) $-7 \times 7$ surface was first obtained by standard flashing of the Si sample ( $n$-type, $\rho \approx 2 \Omega \cdot \mathrm{cm}$ ). And $1 / 3 \mathrm{ML} \mathrm{Ga}$ (in terms of the atomic density of the $\mathrm{Si}(111)$ plane) atoms were evaporated on the $\mathrm{Si}(111)-7 \times 7$ surfaces at room temperature (RT) followed by annealing to $500{ }^{\circ} \mathrm{C}$ for $2 \mathrm{~min}$. This yields the $2 \mathrm{D}$ semiconducting $\sqrt{3} \times \sqrt{3}$-Ga layer on bulkterminated $\mathrm{Si}(111)-1 \times 1$ substrate. ${ }^{8} \mathrm{Ag}$ films were deposited on the $\sqrt{3} \times \sqrt{3}$-Ga surface at RT (flux $0.42 \mathrm{ML} / \mathrm{min}$ in terms of the atomic density of the $\mathrm{Ag}(111)$ plane). The as-prepared samples were studied by STM/STS at $77 \mathrm{~K}$ with a Pt-Ir tip. The $\mathrm{dI} / \mathrm{dV}$ spectra were acquired using lock-in detection of the tunnel current by adding a $30 \mathrm{mV}_{\mathrm{rms}}$ modulation at $1 \mathrm{k}$ $\mathrm{Hz}$ to the sample bias.

On the $\sqrt{3} \times \sqrt{3}-\mathrm{Ga}$ surface, each $\mathrm{Ga}$ adatom is bonded to three $\mathrm{Si}$ atoms of a bulk-truncated $\mathrm{Si}(111)$. As all the $\mathrm{Si}$ dangling bonds are saturated, the surface is semiconducting. ${ }^{9}$ In typical empty state STM images [Fig. 1(a)], there are many random dark sites that are substitutional Si adatoms. The Si adatom has an extra electron and thus may act as donor in this 2D semiconductor system. ${ }^{10}$ Indeed, we found that in the STM images taken at RT, each Si dopant is surrounded by a circular bright zone. On the other hand, when we cooled the sample to $77 \mathrm{~K}$, the contrasts around Si ada-
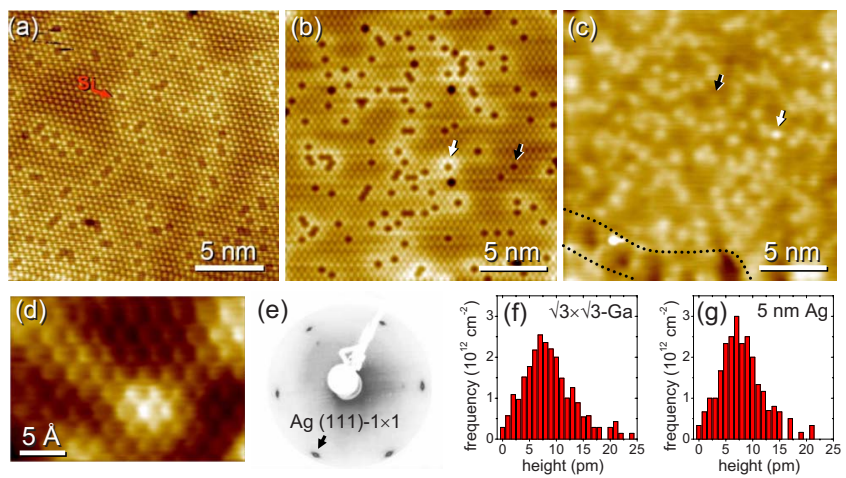

FIG. 1. (Color online) (a) and (b) typical empty state STM image of $\mathrm{Si}(111)-\sqrt{3} \times \sqrt{3}-\mathrm{Ga}$ surface at RT and $77 \mathrm{~K}$, respectively. The positively charged Si dopant, appearing as depression in empty state, is denoted by a red arrow in (a). (c) Filled state STM image of $5 \mathrm{~nm} \mathrm{Ag}$ film grown on $\mathrm{Si}(111)-\sqrt{3} \times \sqrt{3}-\mathrm{Ga}$ substrate at RT. The bright and less bright protrusions are denoted by white and black arrows, respectively. The black feature in the bottom-left of the image is due to a small angle grain boundary, which indicates a slight difference in the orientations of neighboring crystal domains, is consistent with the slightly streaky Ag- $1 \times 1$ LEED spots [see Fig. 1(e)]. (d) Atomically resolved STM image of the protrusions in (c). The atomic structure of $\operatorname{Ag}(111)-1 \times 1$ is clearly resolved. (f) and $(\mathrm{g})$ are the corresponding height distributions of the $\mathrm{Si}$ atoms in (b) and the bright protrusions in (c), respectively. 
toms become inhomogeneous: some $\mathrm{Si}$ adatoms still have surrounding bright zones while others have not or less brightness [Fig. 1(b)]. This can only be accounted for if $\mathrm{Si}$ adatoms are only partially ionized at $77 \mathrm{~K}$, while they are fully ionized at RT. The bright zone thus corresponds to a screened Coulomb potential around charged Si dopants. ${ }^{6}$

The growth of Ag on the $\sqrt{3} \times \sqrt{3}-\mathrm{Ga}$ surface at RT results in large scale, atomically flat $\operatorname{Ag}(111)-1 \times 1$ films, as shown in Figs. 1(c) and 1(e). Surprisingly, many protrusions were observed on top of the Ag films. Statistical analysis gives a ratio of $0.958 \pm 0.050$ for the density of these protrusions versus that of Si dopants in $\sqrt{3} \times \sqrt{3}$-Ga layer. In addition, at RT all protrusions appear identical, whereas at $77 \mathrm{~K}$ they appear differently. The statistical height distribution of the protrusions at $77 \mathrm{~K}$ is plotted in Fig. $1(\mathrm{~g})$, which is very similar to that of Si dopants at $77 \mathrm{~K}$ [Fig. 1(f)].

In order to understand the origin of these protrusions, one should note that the growth of Ag-1 $\times 1$ film does not cause a change to the $\sqrt{3} \times \sqrt{3}-\mathrm{Ga}$ surface. This was confirmed by annealing a fully covered $\mathrm{Ag}$ films to about $100{ }^{\circ} \mathrm{C}$. The film broke into disconnected $\mathrm{Ag}$ islands. In the area among $\mathrm{Ag}$ islands, we observed the $\sqrt{3} \times \sqrt{3}-\mathrm{Ga}$ substrate as well as the $\mathrm{Si}$ adatoms whose density remains about the same as that of a clean $\sqrt{3} \times \sqrt{3}$-Ga surface. Therefore, the protrusions cannot be $\mathrm{Si}$ atoms segregated to the $\mathrm{Ag}$ surface or into the film. In fact, in the atomically resolved STM image as shown in Fig. 1(d), the bright protrusion is superimposed on the Ag atomic lattice instead of locating on a particular lattice site. This is not consistent with an adsorbate or defect on the surface, but is more like a localized potential fluctuation. Combining the above observations and the fact that the density of the surface protrusions is the same as that of Si dopants in the $\sqrt{3} \times \sqrt{3}$-Ga layer, we suggest that the protrusions are indeed images of the interfacial Si dopants.

To understand how STM can image buried Si dopants, we note that the STM image of the dopants is quite sensitive to the tip bias. The protrusions are nearly invisible at the bias below $-0.2 \mathrm{~V}$, where the geometric effect is believed to dominate. ${ }^{11}$ Therefore, the protrusions should have a purely electronic origin. We note the capability of STM to image buried objects through metal films, ${ }^{11-13}$ with the quantized electrons in metal films. The vertically confined electron waves carry the interface information in terms of the reflection phase shift at the interface. As illustrated in Fig. 2(a), the screened Coulomb potential at interface can locally modifies the reflection phase shift in the electron wave, leading to a lateral charge density modulation on top of the Ag films, which is then detected as dopant image by STM. In fact, when we grow Ag films on the clean $\mathrm{Si}(111)-7 \times 7$ surface, on top of the Ag film surface we can see very sharp $7 \times 7$ superlattice, resembling the $7 \times 7$ reconstruction at the interface, which clearly demonstrates the possibility of imaging interface objects under the Ag films with high spatial resolution, in the similar way as the well demonstrated $\mathrm{Pb}$ films on $\mathrm{Si}(111) .^{11-13}$

The phase shift in quantized electron waves can be verified by probing the lateral variation in the energetic position of the quantum well states (QWS). ${ }^{11,13}$ Figure 2(b) shows an isolated Si dopant image. A series of $d \mathrm{I} / d \mathrm{~V}$ spectra were taken along positions away from the center, as shown in
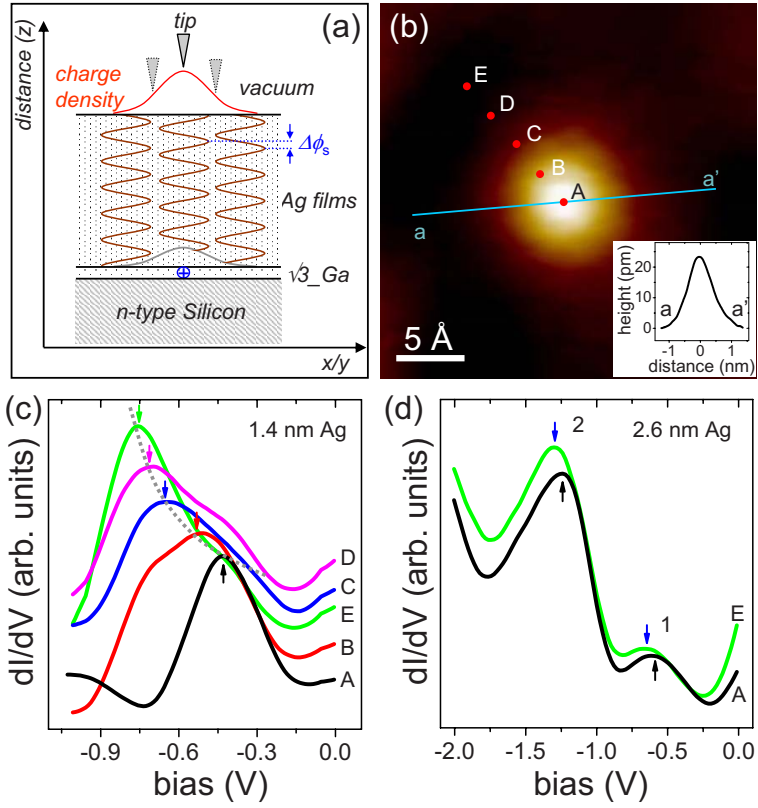

FIG. 2. (Color online) (a) Schematics showing how the dopant Coulomb potential can be imaged on top of the Ag films by the vertically confined electrons. Note that the lateral phase shift induced modulation of the surface charge density resembles the screened Coulomb potential at the interface. (b) An isolated dopant imaged on the Ag films surface. The inset shows the corresponding height profile. (c) and (d) tunneling spectra of 1.4 and $2.6 \mathrm{~nm} \mathrm{Ag}$ films, respectively, obtained along the radial direction of the dopant image at positions equally spaced from point $\mathrm{A}$ to $\mathrm{E}$, as shown in (b). The peaks of the QW resonances are indicated by arrows. The peak shift in (c) is guided for eyes by a dot line.

Figs. 2(c) and 2(d) for two films thicknesses, 1.4 and $2.6 \mathrm{~nm}$, respectively. In both cases prominent QWS were resolved, the positions agreeing well with that in Ref. 14. Away from the center, the QW peaks shift to lower energies, reaching $300 \mathrm{meV}$ for the $1.4 \mathrm{~nm}$ film [Fig. 2(c)]. For thicker film (2.6 nm), two QW peaks (marked by 1 and 2) can be observed, which shift by 40 and $60 \mathrm{meV}$, respectively. The observed QW peak shift $\Delta E$ is related to the phase shift at the interface by: $\Delta E=\frac{d E\left(k_{z}\right)}{d k_{z}} \Delta k_{z}=\frac{d E\left(k_{z}\right)}{d k_{z}} \frac{\Delta \varphi}{2 d}$, where $E\left(k_{z}\right)$ is the energy dispersion of bulk $\mathrm{Ag}$ along $\Gamma-L$ direction $([111]), d$ is the films thickness, and $\Delta \phi$ the lateral phase shift around Si adatoms at the interface. ${ }^{13}$

In the following, we try to extract the screening length at the interface from our experimental data. Figure 3 is a schematic band diagram of $\mathrm{Ag} / \mathrm{Si}$ interface, in which the metalinduced gap states are not included for simplicity. ${ }^{15}$ A depletion region is expected to form near the semiconductor surface due to the electrons flowing from the semiconductor to metal surface. Within a limited energy range near the valence band maximum of $\mathrm{Si}-\sqrt{3} \times \sqrt{3}-\mathrm{Ga}$ surface (denoted by $E_{0}$ as shown in Fig. 3), the energy-dependence of the phase shift in the quantized electron at the interface can be described by: ${ }^{16} \phi_{s}(x, y)=A+B \sqrt{E-E_{0}} \cdot \Theta\left(E-E_{0}\right)$, where $A, B$ are constants, and $\Theta\left(E-E_{0}\right)$ is the Heaviside function. The local band edge shift induced by the dopant potential $\Delta V(x, y)$ (see the red dot line in Fig. 3) will lead to a lateral variation in the phase shift: $\Delta \phi_{s}(x, y)$. Assuming that 


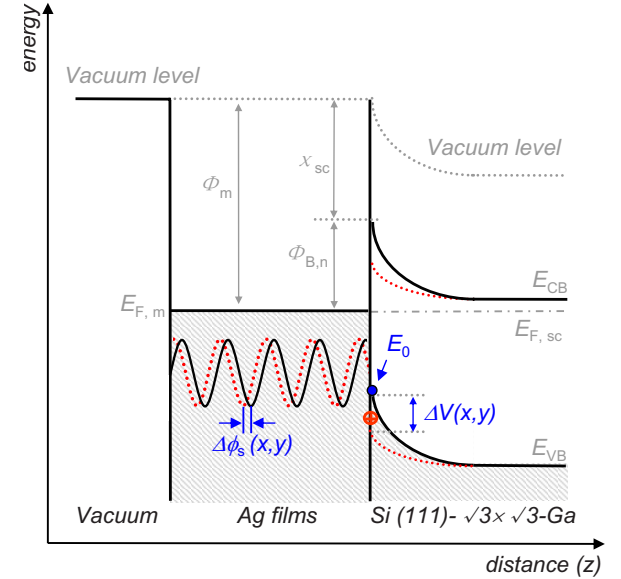

FIG. 3. (Color online) Schematics of the $\mathrm{Ag} / \mathrm{Si}$ interface. $E_{0}$ is the valence band maximum of $\mathrm{Si}-\sqrt{3} \times \sqrt{3}-\mathrm{Ga}$ surface. The local band edge shift induced by the interfacial dopant potential is denoted by $\Delta V(x, y)$ (see the red dot line), which is equivalent with the variation in the Schottky barrier height $\left(\phi_{B, n}\right)$. The resulting reflection phase shift in the quantized electrons is denoted by $\Delta \phi_{s}(x, y)$.

$\Delta V(x, y)$ is only a small perturbation, we then have

$$
\Delta \phi_{s}(x, y) \approx \frac{B}{2} \cdot \frac{\Delta V(x, y)}{\sqrt{E-E_{0}}} .
$$

Therefore, the lateral distribution of the interfacial phase shift resembles the spatial distribution of the interfacial screened potential when the potential is weak.

In order to quantitatively derive the screening length, we need to correlate the height profile in the constant current STM image, $s(x, y)$, with the screening potential $\Delta V(x, y)$. For QWS denoted by $k_{z}$, if its phase shift is changed by $\Delta \phi_{s}(x, y)$ at the interface, the corresponding variation in the charge density on surface, to the first order approximation, is proportional to $\sin ^{2}\left[k_{z} d+\Delta \phi_{s}(x, y)\right]-\sin ^{2}\left(k_{z} d\right)$ $\approx \Delta \phi_{s}(x, y) \sin \left(2 k_{z} d\right)$, where $d$ is the film thickness. At the sample bias $V$, the modulated surface charge density $\Delta I(x, y, V, d)$ is then the summation of all available quantized states: ${ }^{11}$

$$
\begin{aligned}
\Delta I(x, y, V, d) & \propto \int_{E_{F}-\mathrm{eV}}^{E_{F}} \Delta \phi_{s}(x, y) \sin \left(2 k_{z} d\right) d E \\
& =\Delta V(x, y) \frac{B}{2} \int_{E_{F}-\mathrm{eV}}^{E_{F}} \frac{\sin \left(2 k_{z} d\right)}{\sqrt{E-E_{0}}} d E \\
& =\Delta V(x, y) \Re(V, d)
\end{aligned}
$$

where $\mathfrak{R}(V, d)=\frac{B}{2} \int_{E_{F}-\mathrm{eV}}^{E_{F}} \frac{\sin \left(2 k_{2} d\right)}{\sqrt{E-E_{0}}} d E$.

The tunneling current can be related to the tip-sample distance by $I=I_{0}(V) \exp [-2 \kappa s(x, y)]$, where $I_{0}(V)$ the bias dependent surface electron density of states, $s(x, y)$ tip-sample distance and $\kappa=\sqrt{\frac{2 m}{\hbar^{2}} B+\left|k_{\|}\right|^{2}} \cdot{ }^{17}$ In the presence of a small perturbation $\Delta I(x, y, V, d)$, in the constant current mode the tip-sample distance $s$ will response to this perturbation by $\Delta s$
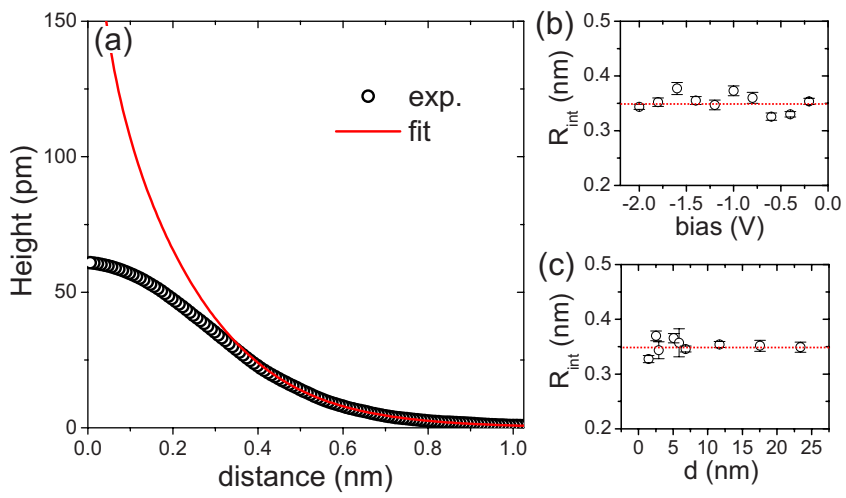

FIG. 4. (Color online) (a) The experimental height profile across one single dopant image on Ag films of $1.4 \mathrm{~nm}$ thick. The solid red line is a fit by Eq. (5). (b) and (c) the interface screening lengths $R_{\text {int }}$, extracted by fitting procedures, as functions of the sample bias $V$ and the film thickness $d$, respectively. The weighted averages of screening lengths are indicated by the horizontal dot red line.

to maintain the tunneling current constant such that: $I=\left[I_{0}(V)+\Delta I(x, y, V, d)\right] \cdot e^{-2 \kappa[s(x, y)+\Delta s(x, y)]}$. Then, we can get

$$
\Delta s(x, y)=\frac{1}{2 \kappa} \ln \left[1+\frac{\Delta I(x, y, V, d)}{I_{0}(V)}\right] .
$$

In analogy to the 2D screening potential on the surface, we assume that the 2D interfacial screened Coulomb potential at small and intermediate distance $r$ can be approximated by $^{18}$

$$
\Delta V(x, y)=\frac{Q}{4 \pi \varepsilon_{0} \varepsilon_{\mathrm{int}}} \frac{1}{r} \exp \left(-\frac{r}{R_{\mathrm{int}}}\right) .
$$

Where $r=\sqrt{x^{2}+y^{2}}, Q$ the dopant charge, $\varepsilon_{0}$ the vacuum permittivity, $\varepsilon_{\text {int }}$ the average of the dielectric constant of interface, and $R_{\text {int }}$ the interfacial screening length. Combined Eqs. (2)-(4), finally, we come to

$$
\Delta s(r)=\frac{1}{2 \kappa} \ln \left[1+\alpha \cdot \frac{1}{r} \exp \left(-\frac{r}{R_{\text {int }}}\right)\right],
$$

where $\alpha=\frac{Q}{4 \pi \varepsilon_{0} \varepsilon_{\text {in }}} \frac{\Re(V, d)}{I_{0}(V)}$.

Equation (5) enables us to fit the experimental STM height profile across a single dopant to obtain the interface screening length $R_{\text {int }}$. The red line in Fig. 4(a) is such a fit, where $\alpha$ and $R_{\text {int }}$ being the fitting parameters. $\kappa$ is equal to $0.012 \mathrm{pm}^{-1}$ calculated with the work function of $\mathrm{Ag}$ and Pt-Ir, assuming that the main contribution to the tunneling current arises from the states near the $\bar{\Gamma}$ point. The fitting curve agrees nicely with the experimental data at the tail of the height profile, but the deviation increases near the dopant center, which is understandable since the above model is valid only when $\Delta V(x, y)$ can be considered as a small perturbation. At the region where dopant potential diverges rapidly to infinity, the model cannot be applicable anymore.

By the above procedure, we have extracted $R_{\text {int }}$ from measurements for various film thicknesses and tip bias, as shown in Figs. 4(b) and 4(c). The thus obtained $R_{\text {int }}$ $=0.35 \pm 0.05 \mathrm{~nm}$ is almost independent of the tip bias and 
the film thickness, agreeing with Eq. (5). It is worthy to note that the sharpness of the tip, i.e., the tunneling disk size, may affect the determination of the screening length. Thus the experimentally extracted screening length should be tipdependent. Such a tip-dependent variation in the screening length can be roughly estimated from Fig. 4(c), in which the screening length obtained by different tips exhibits a small fluctuation within $0.1 \mathrm{~nm}$.

$R_{\text {int }}$ can be derived by a semimicroscopic model for the charge electrostatic potential at metal/semiconductor interface. Assuming the region $z>0$ to be the Ag film, and $z<0$ the bulk $\mathrm{Si}$, the $z=0$ plane defines the $\mathrm{Ag} / \mathrm{Si}$ interface, where a 2D electron gas is inserted to model the $\sqrt{3} \times \sqrt{3}-$ Ga layer. We first start with a charge $Q$ located at a distance $z_{0}$ within the semiconductor below the metal, and then take the limit $z_{0} \rightarrow 0$ to get the potential at the metal/ semiconductor interface. Based on Poisson equations and Thomas-Fermi approximation, together with the corresponding boundary conditions, ${ }^{18}$ we found that the interface Coulomb potential can be approximately expressed as a form of screened Coulomb potential: $V_{s}(r) \approx \frac{Q}{4 \pi \varepsilon_{0} \varepsilon_{\text {int }}} \frac{1}{r} \exp \left[-\frac{r}{R_{\text {int }}}\right]$, with $R_{\text {int }}=1 / \sqrt{\frac{1}{R_{s}^{2}}+\frac{1}{R_{m}^{2} \varepsilon_{r}^{2}}+\frac{1}{R_{2 d}^{2}}}$ the interface screening length we are interested in. $R_{s}, R_{m}$, and $R_{2 d}$ are the screening lengths of bulk semiconductor, bulk metal and 2D electron gas, respectively, and $\varepsilon_{r}$ is the dielectric constant of semiconductor $\left(\varepsilon_{r}=11.5\right.$ for $\left.\mathrm{Si}\right)$. As our $\mathrm{Si}$ sample is lowly doped $\left(n=2.3 \times 10^{15} \mathrm{~cm}^{-3}\right)$, we can take the limit $1 / R_{s} \rightarrow 0$. Employing $R_{m}=0.06 \mathrm{~nm}$ for $\mathrm{Ag}$ (Ref. 1) and $R_{2 d}=2.1 \pm 0.6 \mathrm{~nm}$ experimentally obtained by fitting the height profiles across the $\mathrm{Si}$ dopants in clean $\sqrt{3} \times \sqrt{3}-\mathrm{Ga}$ surface at $77 \mathrm{~K}$, we obtain $R_{\text {int }}=0.65 \pm 0.07 \mathrm{~nm}$.

The agreement between the theoretical and experimental value, $0.35 \pm 0.05 \mathrm{~nm}$, is very good regarding the fact that we neglected the microscopic details at the interface upon the formation of the metal-semiconductor junction, e.g., the presence of the extra electrons at the interface contributed by the semiconductor (see the depletion region in Fig. 3), which may further reduce the interface screening length. Finally, our result shows that the metal/semiconductor interfacial screening length $\left(\times 10^{-10} \mathrm{~m}\right)$ is about one order of magnitude smaller than the typical screening length in bulk semi- conductor $\left(\times 10^{-9} \mathrm{~m}\right)$, but still one order of magnitude larger than that in bulk metal $\left(\times 10^{-11} \mathrm{~m}\right)$. This result thus provides an important reference for the design of the future chargebased quantum electronic devices.

Finally, we noticed that the lateral resolution in the present study, and the fact that $R_{\text {int }}$ shows little change with increasing film thickness, cannot be solely ascribed to the large anisotropy of the in-plane $\left(m_{\|}\right)$and transversal $\left(m_{\perp}\right)$ effective masses $\left(m_{\|} / m_{\perp}\right.$ is only 2-3 for Ag), as revealed for the case of $\mathrm{Pb} .{ }^{13}$ Considering the STM tunneling mechanism, an electron moving at around the Fermi level has a exponential decay constant $\kappa=\sqrt{\frac{2 m}{\hbar^{2}} B+\left|k_{\|}\right|^{2}}$, implying that the electron which moves perpendicularly toward the surface (small $k_{\|}$) has a relatively large tunneling probability. ${ }^{17}$ This focusing effect is further amplified by the fact that the transmission probability is biggest for the electrons traversing along the normal direction, since the effective barrier width is smallest. The electrons with nonzero parallel kinetic energy will encounter much larger effective barrier, thus much smaller transmission probability, especially under the case of atomically sharp tip. Therefore, the dopant image detected by STM is primarily contributed by the electrons limited within a very narrow $k_{\|}$range, leading to the almost thicknessindependent lateral resolution.

In conclusion, we report the direct observation of the screening potential around individual dopants at the metal/ semiconductor interface with STM/STS, and experimental measurement of the interface screening length. It was verified that the dopants imaged on top of the metal film surfaces are a mapping of the interface screened Coulomb potential by the quantized electrons. The experimentally extracted interface screening length agrees well with the theoretical value we derived from a semimicroscopic model. Our work is helpful for the understanding of the physics of screening at interfaces, which is of basic importance for the design of devices such as surface plasmonic waveguides, MOS devices and so on.

We thank Yukio Hasegawa for stimulating discussions and comments. This work was supported by the National Science Foundation (Grant No. 10874210), and MOST (Grant No. 2007CB936800) of China.

\footnotetext{
*Corresponding author; yjiang3@uci.edu

${ }^{1}$ N. W. Ashcroft and N. D. Mermin, Solid State Physics (Saunders College, Philadelphia, 1976).

${ }^{2}$ D. S. Chemla and J. Shah, Nature (London) 411, 549 (2001).

${ }^{3}$ P. Zhang et al., Phys. Rev. Lett. 98, 067005 (2007).

${ }^{4}$ M. F. Crommie et al., Science 262, 218 (1993); Y. Hasegawa and P. Avouris, Phys. Rev. Lett. 71, 1071 (1993).

${ }^{5}$ M. Ono et al., Phys. Rev. Lett. 96, 016801 (2006).

${ }^{6} \mathrm{Ph}$. Ebert, Surf. Sci. Rep. 33, 121 (1999) and references therein.

${ }^{7}$ J. Tersoff and D. R. Hamann, Phys. Rev. Lett. 50, 1998 (1983); Phys. Rev. B 31, 805 (1985).

${ }^{8}$ J. Nogami et al., Surf. Sci. 203, L631 (1988).

${ }^{9}$ J. M. Nicholls et al., Phys. Rev. B 35, 4137 (1987).

${ }^{10}$ S. Landrock et al., Appl. Phys. Lett. 95, 072107 (2009).
}

${ }^{11}$ W. B. Jian et al., Phys. Rev. Lett. 90, 196603 (2003); S. M. Lu et al., Phys. Rev. B 75, 113402 (2007).

${ }^{12}$ H. B. Yu et al., Appl. Phys. Lett. 81, 2005 (2002).

${ }^{13}$ I. B. Altfeder et al., Phys. Rev. Lett. 80, 4895 (1998); I. B. Altfeder et al., ibid. 88, 206801 (2002).

${ }^{14}$ C. S. Jiang et al., Phys. Rev. B 64, 235410 (2001).

${ }^{15}$ R. T. Tung, Phys. Rev. Lett. 84, 6078 (2000).

${ }^{16}$ M. A. Mueller et al., Phys. Rev. B 40, 5845 (1989).

${ }^{17}$ L. E. C. van de Leemput and H. van Kempen, Rep. Prog. Phys. 55, 1165 (1992).

${ }^{18}$ M. Krčmar et al., Phys. Rev. B 61, 13821 (2000); M. R. Krčmar and W. M. Saslow, ibid. 66, 235310 (2002); A. Laubsch et al., ibid. 80, 245314 (2009). 